\title{
Revisão integrativa sobre intolerância religiosa em povos de matriz africana
}

\author{
Integrative review on religious intolerance in african matrix people \\ Revisión integrativa sobre la intolerancia religiosa en las personas de la matriz africana
}

Recebido: 20/08/2021 | Revisado: 29/08/2021 | Aceito: 08/09/2021 | Publicado: 10/09/2021

\author{
Ana Carolina Monteiro dos Santos de Alcântara \\ ORCID: https://orcid.org/0000-0002-7859-3542 \\ Universidade Federal do Pará, Brasil \\ E-mail: acarolinams.adv@gmail.com \\ Maria de Nazareth Rodrigues Malcher de Oliveira Silva \\ ORCID: https://orcid.org/0000-0003-4405-7378 \\ Universidade de Brasília, Brasil \\ E-mail: malchersilva@unb.br
}

\begin{abstract}
Resumo
Historicamente, a intolerância religiosa é um fenômeno presente no cenário mundial, marcado por um conjunto complexo de significados atribuídos as experiências discriminatórias, que em sua maioria são praticados contra grupos minoritários. Este estudo buscou conhecer a literatura nacional e internacional sobre a caracterização da intolerância religiosa em povos e comunidades de matriz africanas, pretendendo conhecer este fenômenos, pelas perspectivas das pesquisas científicas, apontando a heterogeneidade, e seus significados. Usou como método o estudo da literatura integrativa e análise de perfil e conteúdo. Os 17 artigos apresentaram um conjunto de significados atribuídos ao fenômeno, com estreita relação entre religião, crenças sociais opressivas e sofrimento psíquico, de modo, que as religiões podem contribuir para o enfrentamento e/ou para o agravamento da intolerância, além de promoverem profundas mudanças sociais, jurídico e legislativas, mostrando como um fenômeno heterogêneo e abrangente.
\end{abstract}

Palavras-chave: Intolerância Religiosa; África; Fenomenologia.

\begin{abstract}
Historically, religious intolerance is a phenomenon present in the world scenario, marked by a complex set of meanings attributed to discriminatory experiences, which are mostly practiced against minority groups. This study sought to understand the national and international literature on the characterization of religious intolerance in peoples and communities of African origin, intending to know this phenomenon, from the perspective of scientific research, pointing out the heterogeneity and its meanings. It used as a method the study of integrative literature and profile and content analysis. The 17 articles presented a set of meanings attributed to the phenomenon, with a close relationship between religion, oppressive social beliefs and psychological distress, so that religions can contribute to confronting and/or worsening intolerance, in addition to promoting profound changes social, legal and legislative, showing how a heterogeneous and comprehensive phenomenon.
\end{abstract}

Keywords: Religious Intolerance; Africa; Phenomenology.

\section{Resumen}

Históricamente, la intolerancia religiosa es un fenómeno presente en el escenario mundial, marcado por un complejo conjunto de significados atribuidos a experiencias discriminatorias, que se practican principalmente contra grupos minoritarios. Este estudio buscó comprender la literatura nacional e internacional sobre la caracterización de la intolerancia religiosa en pueblos y comunidades de origen africano, con la intención de conocer este fenómeno, desde la perspectiva de la investigación científica, señalando la heterogeneidad y sus significados. Se utilizó como método el estudio de la literatura integradora y el análisis de perfil y contenido. Los 17 artículos presentaron un conjunto de significados atribuidos al fenómeno, con una estrecha relación entre religión, creencias sociales opresivas y angústia psicológica, de manera que las religiones pueden contribuir a enfrentar y / o agravar la intolerancia, además de promover profundos cambios sociales, legales y legislativo, mostrando cómo un fenómeno heterogéneo e integral.

Palabras clave: Intolerancia religiosa; África; Fenomenología.

\section{Introdução}

Historicamente, o fenômeno religioso se revelou como um elemento constitutivo importante na subjetividade humana na sociedade, pois produz significados e efeitos concretos na vida das pessoas (Santos, 2014), e que portanto, requer processos 
associados a este fenômeno, como por exemplo, a intolerância religiosa.

A compreensão da intolerância religiosa requer um dialogo com a concepção da tolerância; neste ponto de vista situamos que a Organização das Nações Unidas (1995) compreende tolerância como o ato de respeitar a diversidade cultural, modos de convicções do outro, expressão de diferenças, incluindo a liberdade religiosa; enquanto, para Viegas (2016) o fenômeno da intolerância se evidencia quando esses fatores humanos divergirem no limite de tentar destruí-las, ou quando se recusarem a aceitar os princípios de tolerância e liberdade de outras pessoas ou grupos. Com este entendimento temos que, a partir das rupturas nas práticas da tolerância religiosa, a intolerância se tornou parte da política colonizadora e da formação histórico social de algumas sociedades, como a brasileira, na qual a adoção de uma religião oficial comprometeu liberdades individuais e coletivas (Guilliomet, 2020), representando graves violações aos direitos humanos de grupos minoritários, como povos e comunidades tradicionais de matriz africana, se diferenciam de um grupo dominante por se auto considerarem ou serem considerados diferentes em fatores como nacionalidade, cultura, tradição, língua e religião (Gonçalves, 2011).

O período colonial da sociedade brasileira se formou pela introdução da cultura europeia, das etnias nativas que aqui já habitavam, além dos grupos étnicos africanos trazidos para o Brasil, por meio do lucrativo tráfico, reconhecido diáspora africana (Prandi, 2000), ocasionando, portanto, neste período uma diversidade de etnias africanas ${ }^{1}$ na cultura.

Gaia, Da Silva \& Scorsolini (2021) afirmam que definir as matrizes africanas existentes no Brasil é uma tarefa árdua, acurada sobre as singularidades do patrimônio ancestral, cultural, religioso, de saberes e tradições africanas dos grupos etnicos africanos, no contexto diaspórico brasileiro, pela ausência da padronização até em uma mesma matriz, em virtude, entre outros fatores da junção entre grupos de nações próximas e de diferentes matriz, resultando dificuldade de se estabelecer um perfil coeso sobre $\mathrm{o}$ assunto.

Nogueira (2020) reconhece as Comunidades Tradicionais de Terreiro (CTTro) como todas as práticas afro-brasileiras, religiões de matriz africana e tradições afro-brasileiras. Portanto, o negro, por meio das religiões de matriz africana se firmou nos terreiros, verdadeiros elos de continuidade africana que funcionam também como centros de organização política, afirmação identitária e resistência cultural (Sodré, 2019; Nascimento, 2020).

Assim, as práticas afro-brasileiras por um lado mostram-se representadas significativamente na população, por exemplo no resultado do censo brasileiro onde 3\% dos brasileiros se declararam afro religiosos, pertencentes as matrizes das umbandas e candomblés. Entretanto, por outro lado observou-se neste censo apenas duas únicas opções oferecidas na pesquisa para a categoria, ignorando a diversidade da matriz africana, tratando com homogeneidade (Instituto Brasileiro de Geografia e Estatística- IBGE, 2010) $)^{2}$.

Este fato simboliza o desenvolvimento histórico na qual africanos e seus descendentes sofreram repressões por motivos religiosos, impedimentos ao exercício de suas práticas, condenações inquisitoriais, perseguições e processos criminais, que atravessaram os séculos XVIII e XIX, sob o discurso da moral em oposição ao ético ou inclusivo (Araújo, Acioly, Costa \& Gomes, 2016), gerador de espaço secundário na sociedade (Nascimento, 2019; Prandi, 2000), e alvo da intolerância religiosa no Brasil, segundo dados do Relatório sobre Intolerância e Violência Religiosa (Brasil, 2015)³.

\footnotetext{
${ }^{1}$ Este estudo destaca como etnias africanas com base aos estudos de Law (2006) como iorubás, fons, mahis, hauçás, éwés, ashantis, congos, quimbundos, umbundos, macuas, lundas que apresentaram diversos valores com o sagrado, nas quais foram difundidas em religiosidades e religiões denominadas de matriz africana em diferentes regiões brasileira. Diante disso, considera-se como as tradições religiosas de matrizes africanas destacadas no Brasil os candomblés, as umbandas, os congados, os xangôs, os batuques, o tambor de mina, cultos a ifá, pajelanças, juremas, catimbós (Da Motta, 2006; Munanga \& Gomes, 2016; Sodré, 2019), que seguem formas de organização social, uso próprio da terra e dos recursos naturais, manifestação cultural, social, religiosa, ancestral, econômica, transmissão de conhecimentos e práticas, além da formação de territórios particulares caracterizados pela vivência comunitária e prestação de serviço comunitário (Brasil, 2007; 2013).
}

${ }^{2}$ IBGE. Censo. Censo Populacional 2010. https://biblioteca.ibge.gov.br/visualizacao/periodicos/94/cd_2010_religiao_deficiencia.pdf

${ }^{3}$ BRASIL. Relatório sobre Intolerância e Violência Religiosa. Disponível em: https://www.gov.br/mdh/pt-br/acesso-a-informacao/participacaosocial/cnrdr/pdfs/relatorio-de-intolerancia-e-violencia-religiosa-rivir-2015/view. 
Entre diversas formas de intolerância Nogueira (2020), destaca "na perseguição, somos todos "macumbeiros" - no sentido negativo da palavra -, por isso é preciso que nos vejamos todos como irmãos e parte de uma cultura com gênese comum" (p. 15). Com isso, Do Nascimento (2016) destaca que não à toa que estudiosos, ativistas e movimentos sociais passaram a adotar o termo racismo religioso para designar as práticas de intolerância religiosa utilizadas como estatégias de perpetuação do sistema de desigualdades e manutrenção de privilégios de um grupo religioso sobre outro, fortalecendo o sistema de garantia de direitos das classes religiosas afetadas, pois a organização dos coletivos sociais encontram a potência de transformação social.

O movimento negro a nível internacional e nacional empreendeu esforços para promover pautas de inclusão étnico-racial e cumprimento de metas para a diminuição de desigualdades e superação do racismo (Brasil, 2009), que enfocou na elaboração de leis e implantação de políticas públicas. Barreto (2017) destaca que essas mobilizações compõem formas particulares psíquicos produtores de sentido social, e que Nascimento (2020) ressalta fruto de um pensamento decolonial afrocentrado.

No entanto, apesar de conseguirem algumas respostas as suas reivindicações, a intolerância religiosa contra adeptos de religiões de matrizes africanas ainda são atuais e com manifestações agressivas e danosas, pois, em geral, "atingem uma coletividade de forma concreta e subjetiva, fragilizando a integridade da segurança e do sagrado ao mesmo tempo" (Mata, 2015, p.08), e de opressão sistematizada de um povo, frente a visão marginalizada do negro ao longo dos tempos na sociedade (Fanon, 2010).

\section{Metodologia}

Este estudo deriva da pesquisa de mestrado acadêmico realizada pela primeira autora na Universidade Federal do Pará, Pós-graduação em Psicologia, sob orientação da segunda autora. Trata-se de revisão integrativa, em busca de uma ampla compreensão da literatura com abrangência das conclusões e reflexões em relação a temática do estudo. Souza et al (2010) destacam que no estudo integrativo da literatura o pesquisador deve percorrer seis etapas, que vão desde a elaboração de uma pergunta norteadora, pesquisa em bases de dados, extração dos dados, análise, discussão e apresentação dos dados.

Neste sentido, a questão norteadora de pesquisa foi "qual a caracterização na literatura da intolerância religiosa contra povos e comunidades de matriz africana?”. Inicialmente, foi realizado pesquisa em Descritores em Ciências da Saúde (DECS/MESH) e localizado os termos "Intolerância", "Religião" e "África", que para traçar um panorama mais amplo de resultados sobre o assunto foram utilizados como termos centrais, mas separados com parenteses e moderadores booleanos and e seus sinônimos com or, e depois foram aplicados nas bases de dados, Academic Search Premier - ASP (EBSCO), Biblioteca Virtual em Saúde (BVS), Cambridge Core, Portal de Periódicos Capes, Scientific Eletronic Library Online (SciELO), Science Direct e Scopus.

A busca dos artigos ocorreu no período de setembro a dezembro de 2020, com critério de inclusão artigos científicos com textos completos teóricos e empíricos, sem tempo de publicação, nos idiomas português e inglês; e como critério de exclusão textos incompletos, repetidos nas bases de dados e que tratavam de temáticas de outras religiões. A seleção dos artigos ocorreu primeiro por um dos descritores no título e segundo pela leitura no resumo sobre a temática da pesquisa.

A busca inicialmente apresentou 895 nas bases de dados selecionadas, nos quais foram aplicados os critérios de inclusão, finalizando na seleção dos 17 artigos, como ilustrado na Figura 1, apresentando o quantitativo encontrado e selecionado por cada base de dados. 
Figura 1 - distribuição do quantitativo dos artigos encontrado e selecionado por cada base de dados.
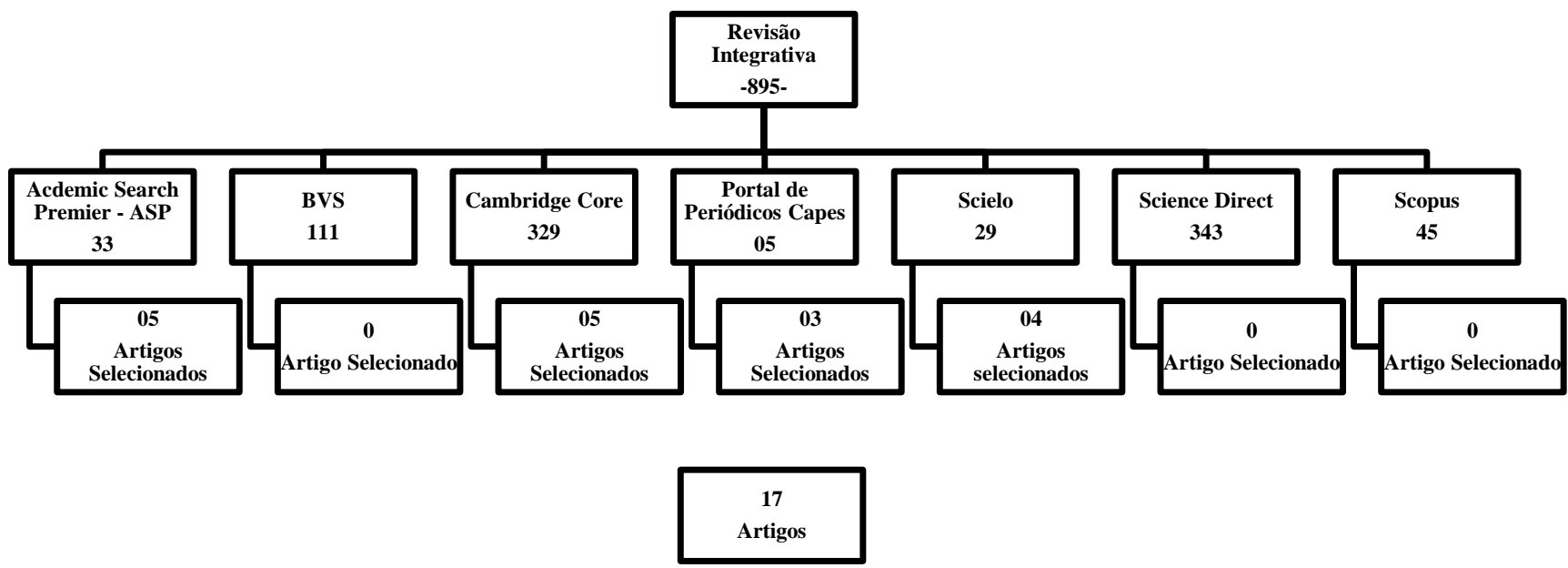

Fonte: Pesquisa (2021).

Os dados dos 17 artigos foram extraídos e tratados em duas planilhas temáticas: (1) sobre o perfil da literatura, com as variáveis, tipos de periódicos, áreas de estudo, tipos de estudo, estratégias de estudo, ano, estratégias de análise de dados, cenários, sujeitos das pesquisas, conteúdo, principais resultados, citações relevantes, conceitos relevantes e referências; e (2) sobre o conteúdo, com as variáveis, contexto sobre o tema, conceitos relevantes sobre o tema, caracterização, contextos religiosos encontrados, narrativas, abordagens teóricas.

A análise realizada foi de frequência numérica simples nos dados de perfil da literatura e temático integrativo no conteúdo dos dados, por meio de levantamento das similaridades, distinções e especificidades do conteúdo apresentado, para assim compreender a integralidade da literatura estudada sobre o tema da intolerância religiosa em povos e comunidades de origem africana.

\section{Resultados e Discussão}

Inicialmente o perfil da literatura dos 17 artigos selecionados sobre a temática intolerância religiosa em povos e comunidades de matriz africana apresentaram uma diversidade de dados organizados na Tabela1.

Tabela 1 - Perfil da literatura dos estudos selecionados.

\begin{tabular}{lll}
\hline Conteúdos & Características & $\mathbf{N = 1 7 ( 1 0 0 \% )}$ \\
\hline Anos & Anos 90 & $02(11,7 \%)$ \\
\multirow{3}{*}{ Continente } & Anos 2000 & $15(88,2 \%)$ \\
\cline { 2 - 3 } & Africano & $05(29,4 \%)$ \\
\multirow{2}{*}{ Áreas dos estudos } & Americano & $08(47,0 \%)$ \\
& Europeu & $04(23,5 \%)$ \\
\cline { 2 - 3 } Tipos de Estudo & Humanas & $14(82,3 \%)$ \\
& Saúde & $02(11,7 \%)$ \\
& Educação & $01(05,8 \%)$ \\
\cline { 2 - 3 } & Estudo Empírico & $15(88,2 \%)$ \\
& Estudo Teórico & $02(11,7 \%)$ \\
\cline { 2 - 3 }
\end{tabular}




\begin{tabular}{lll}
\cline { 2 - 2 } Cenários do estudo & $\begin{array}{l}\text { Estudantes universitários } \\
\text { Grupos étnicos raciais e religiosos }\end{array}$ & $05(29,4 \%)$ \\
Abordagem dos Estudos & Estudos Qualitativos & $12(70,5 \%)$ \\
\cline { 2 - 3 } & Estudos Mistos & $07(41,1 \%)$ \\
\cline { 2 - 3 } Estratégias de pesquisa & Entrevistas & $10(58,8 \%)$ \\
& Documentos & $06(35,2 \%)$ \\
& Mídias televisivas & $01(05,8 \%)$ \\
\hline
\end{tabular}

Fonte: Pesquisa (2021).

O perfil da literatura mostrou um predomínio de publicações nos anos 2000 (88,2\%), no continente Americano norte (47,0\%), na área das humanas (82,3\%), com pesquisas qualitativas $(58,8 \%)$ empíricas $(88,2 \%)$, com grupos étnicos raciais e religiosos, utilizando estratégias de entrevistas $(58,8 \%)$.

Estudos sobre intolerância religiosa prevaleceram em periódicos nas ciências humanas, nos campos da psicologia (41,1\%), e 29,4\% da religião como também da política, compreendendo como uma temática pouco estudada no campo da saúde e da educação. Portanto, percebe-se um aumento significativo de pesquisas qualitativas nos últimos anos, focadas nas vivências de grupos étnicos, raciais e religiosos sobre o fenômeno da intolerância religiosa a nível global, permitindo refletir de forma ampla o fenômeno histórico.

Nos 17 artigos organizamos três categorias integrativas, representando uma abrangência e complexidades do fenômeno: (1) estudos que caracterizam intolerância religiosa nas minorias étnicas, raciais, sexuais e comunidades religiosas, apresentando contextos dos sofrimentos psíquicos; (2) estudos de casos sobre judicialização de litígios religiosos e direito à li,berdade religiosa; e (3) estudos sobre a relação entre conflito político, religião e identidade.

Nos estudos sobre a caracterização da intolerância religiosa foram apresentados diversos aspectos como, níveis e tipos de intolerâncias entendidas como crenças opressivas, afirmando que interferem na construção subjetiva dos sujeitos estudados, sobre aspectos de sexismo, racismo, preconceito sexual, ageísmo, classismo e intolerância religiosa em si (Allport \& Ross, 1967; Eisinga et al., 1999; Aosved \& Long, 2006; Aosved, Long \& Voller, 2009; Doebler, 2015); questões de identidade, diferença, preconceito e religião, levados a apreciação de Tribunais em busca desses posicionamentos sobre a intolerância (Du plessis, 2009; Benson, 2013) e caracterização por meio de estudos teóricos de obras literárias (Beneke \& Grenda, 2011; Sorkin, 2017).

Nascimento (2015) apresentou uma distinção particularizada da intolerância religiosa contra povos e comunidades de matriz africana brasileira, reportando-se a obra literária e televisiva "Tenda dos Milagres", que retratou o contexto religioso do Candomblé na Bahia, apresentando narrativas de religiosos sobre suas experiências intolerantes que ilustram contextos de sofrimentos psíquicos cotidianos motivados pela discriminação e estigma social, e rejeição familiar, gerando tristeza e revolta nos religiosos. O autor discorreu sobre a intolerância religiosa como consequência de um processo histórico de demonização das práticas e rituais desses religiosos, que não se desconstruíram com o tempo, de modo que a maioria de seus adeptos vivem ainda na invisibilidade social e carregam traços e dores de exclusão até os dias atuais. Nesta perspectiva, o produto televisivo mostrouse na perspectiva de difundir as ideias de uma “África oprimida, subordinada e marginalizada” (p. 09), frequentemente, associada à escravidão e a piadas jocosas de cunho discriminatório.

Os estudos sobre a intolerância religiosa como conflito político, religião e identidade religiosa destacaram a necessidade de aprimoramento das leis e da consciência de grupos étnicos minoritários e, de problematizar a compreensão conceitual sobre religião e identidade africana (Osborn, 2014; Sanni, 2016). Concomitantemente, o conceito de intolerância religiosa nos estudos apresentaram sinônimos diversificados como, preconceito, discriminação e estereótipo contra indivíduos, grupos religiosos, religiões ou manifestações de crença e de fé, assemelhada e, geralmente, associada a outras formas de intolerância (Asvoed \& Long, 2006; Crosby \& Varella, 2014); violência religiosa (Osborn, 2014); e por meio de sinônimos de liberdade de religião, 
intolerância racial, rede social étnica, tratamento injusto pela origem étnica, identidade étnica (Du plessis, 2009; Doebler, 2015; Ikram, 2016; Mccleary-gaddy \& Miller, 2019).

Os resultados nos estudos mostram duas categorias integrativas principais relevantes destacadas na Tabela 2 com respectivos autores.

Tabela 2. Resultados.

\begin{tabular}{ll}
\hline Autores (Ano) & Resultados importantes \\
\hline Allport, Ross, 1967 & $\begin{array}{l}\text { Níveis e tipos de intolerância relacionam religião a comportamentos e } \\
\text { crenças opressivas, causadoras de sofrimento psíquico }\end{array}$ \\
Eisinga et al, 1999 & \\
Aosved, Long, 2006 & \\
Aosved, Long, voller, 2009 & \\
Crosby, Varela, 2014 & \\
Osborn, 2014 & \\
Doebler, 2015 & \\
Nascimento, 2015 & \\
Ikram et al, 2016 & \\
Mcewen, Steyn, 2016 & \\
Sanni, 2016 & \\
Ezeh et al, 2017 & A interferência jurídico-legislativas e mudanças legislativas para proteção a \\
Mccleary-gaddy, Miller, 2019 & liberdade religiosa suscitam debates em torno da compreensão sobre a \\
\hline Du plessis, 2009 & coexistência de diferentes valores e culturas. \\
Beneke, Grenda, 2011 & \\
Benson, 2013 & \\
Sorkin, 2017 & \\
\hline
\end{tabular}

Fonte: Pesquisa (2021).

Avaliamos que, os resultados dos estudos apresentaram um contexto ambivalente onde se por um lado a intolerância religiosa mostrou-se como um processo causal de sofrimento psíquico, por outro lado também pode gerar, por meio de interferências jurídicas, percepção de igualdade e de coexistência cultural, promovendo sentido de identificação e coletividade social. Na primeira categoria os estudos mostraram preconceitos voltados às minorias étnica, raciais, sexuais e comunidades religiosas, com discriminação cotidiana, como, consciência do estigma, invisibilidade percebida, hostilidade, incompatibilidade entre visões de mundo, fanatismo, pouco respeito pela vida humana e preocupações existenciais, que acabam por contribuir para instalar processos em saúde mental, como depressão, ansiedade, angústia, pensamento de morte, insegurança, desconfiança, tristeza, vergonha e rejeição.

Na segunda categoria os resultados dos estudos tratam de contextos relacionados por interferência jurídico-legislativas na qual apresentam que a liberdade religiosa suscita debates sobre a coexistência de diferentes valores, culturas, noções de igualdade e entre outras. Além disso, os estudos elencaram diferenças entre as legislações de proteção a liberdade religiosa e as decisões aplicadas pelos tribunais da justiça nos casos envolvendo processos de conflitos religiosos, e afirmando que questões envolvendo identidade, diferenças, preconceitos relacionados a aspectos religiosos contribuem com cenários de conflitosos. (Du plessis, 2009; Beneke \& Grenda, 2011, Benson, 2013, Sorkin, 2017).

Em relação a quem comete a intolerância religiosa diversos estudos relacionam a grupos específicos. Allport e Ross (1967) e Eisinga et al (1999) destacam os religiosos cristãos como grupo de maior preconceito em relação a pessoas não religiosas, e os descrentes; enquanto que Doebler (2015) ressaltou que países mais instáveis política e economicamente e com mais conflitos históricos são significativamente mais propensos a comportamentos intolerantes. Outro aspecto sobre a intolerância é apresentado por Aosved, Long e Voller (2009) na qual referem o nível e tipografia associado a comportamentos e crenças opressivas socialmente instituídas por gênero, nas quais há uma maior adesão entre homens do que entre mulheres para seguir o exemplo 
estas crenças, tanto individualmente quanto entre pessoas do mesmo nível cultural. Por exemplo, a presença desses fatores são preditores da aceitação da ocorrência de estrupo. Estes autores criaram, com base as crenças opressivas, uma ferramenta específica para mensuração, considerando aspectos globais e particulares de cada pessoa.

Entretanto, alguns estudos apontam para estratégias de superação da intolerância religiosa, como Ikram et al (2016) e Ezeh (et al, 2017) que propõem uma reestruturação dos efeitos de incidência de mortalidade e do preconceito etnocultural com o fortalecimento da solidariedade, da cooperação, do senso de pertencimento, da rede social, da religião e da identidade dos grupos minoritários. Mcewen e Steyn (2016) e Sanni (2016) recomendam a aplicação de recursos educacionais inclusivos junto as comunidades religiosas e instituições teleológicas de formação de líderes religiosos para promover debates sobre a diversas formas de pensar conceitos sobre religião e identidade.

Finalmente os estudos apontaram para conteúdos sobre intolerância religiosa com povos e comunidades e matriz africana como uma caracterização diversa de grupos minoritários, que mesmo sendo preponderante em grupos específicos, é um fenômeno independente na sociedade, nucleada sob dois aspectos, de opressão social, geradora de contextos jurídicos, mas também de emoções e até sofrimentos psíquico; e de processo de ressignificação para a liberdade religiosa de aceitação dos diferentes valores, culturas para noções de igualdade.

Observou-se no estudo da literatura uma lacuna em pesquisas de grupos específicos de matriz africana, na qual apenas um estudo pesquisou este fenômeno no contexto do Candomblé (Nascimento, 2015). Portanto, mostra-se relevância o investimento de estudos empíricos, de estratégia fenomenológica, com enfoque específico na vivência, e em grupos ou pessoas. Deste modo, os estudos apontaram a intolerância religiosa com povos e comunidades e matriz africana como uma caracterização diversa de grupos minoritários, que mesmo sendo preponderante em grupos específicos, é um fenômeno independente na sociedade, nucleada sob dois aspectos, de opressão social, geradora de contextos jurídicos, mas também de emoções e até sofrimentos psíquico; e de processo de ressignificação para a liberdade religiosa de aceitação dos diferentes valores, culturas para noções de igualdade.

Finalmente, é importante destacar que os estudos sobre intolerância religiosa possibilitaram compreender o fenômeno da intolerância em diferentes contextos, demonstrando que as causas e efeitos desse fenômeno expressam a ação humana e o mundo vivido em suas singularidades, bem como enfatizam que os conflitos religiosos se relacionam diretamente a comportamentos discriminatórios e sofrimentos psíquicos.

\section{Considerações Finais}

Este estudo buscou conhecer a literatura nacional e internacional sobre a caracterização da intolerância religiosa em povos e comunidades de matrizes africanas, pretendendo apresentar o fenômeno pelas perspectivas das pesquisas científicas, apontando a heterogeneidade, seus significados, e evidenciando a importância desta temática, que invisibilizada historicamente, integra-se cada vez mais a diversas áreas de estudo científico.

A intolerância religiosa mostra-se, portanto, como um fenômeno multifatorial, multidimensional e atual, permeado de significados em torno das experiências discriminatórias, prioritariamente em grupos minoritários étnicos-raciais e religiosos, que agregam contextos singulares, porém complexos, necessários de investimentos em mais estudos que possibilitem mudanças sociais, jurídicas e legislativas no acolhimento da diversidade social.

\section{Agradecimentos}

Agradecemos ao apoio da Linha fenomenologia Teoria e clínica do Programa de Pós-graduação em Psicologia, do Instituto de Filosofia e Ciências Humanas da Universidade Federal do Pará (PPGP/IFCH/UFPA). 


\section{Referências}

Allport, G. W. \& Ross, J. M. (1967). Personal religious orientation and prejudice. Journal of Personality and Social Psychology, v. 5, n. 4, p. 432.

Aosved, A. C. \& Long, P. J. (2006). Co-occurrence of rape myth acceptance, sexism, racism, homophobia, ageism, classism, and religious intolerance. Sex Roles, v. 55, n. 7, p. 481-492.

Aosved, A. C., Long, P. J. \& Voller, E. K. (2009). Measuring sexism, racism, sexual prejudice, ageism, classism, and religious intolerance: The intolerant schema measure. Journal of Applied Social Psychology, v. 39, n. 10, p. 2321-2354.

Araújo, V. A. B. de; Acioly, A. C. (2016). Intolerância contra Afro-Religiosos: conhecendo o candomblé dentro da sala de aula. In.: XVII Encontro Estadual de História, v. 17, n. 1, p. 568-577.

Barreto, R. M. Contribuições psicanalíticas para a compreensão do preconceito racial: um estudo de caso. Robenilson Moura Barreto.-2017.

Beneke, C., Grenda, C. S. (Ed.). (2011). The first prejudice. Religious tolerance and intolerance in early America. University of Pennsylvania Press.

Benson, I. T. (2013). Trabalhos inter-religiosos no Canadá e na África do Sul com foco especial na elaboração de uma Carta sul-africana de Direitos Religiosos e Liberdades. Herv. teol. stud., Pretória, v. 69, n. 1, p. 01-13, jan.

Bicudo, M. A V. (2011). Pesquisa qualitativa segundo a visão fenomenológica. Ed. Cortez.

Brasil. (2013). Plano de Desenvolvimento Sustentável dos Povos e Comunidades Tradicionais de Matriz Africana - Secretaria de Políticas de Promoção da Igualdade Racial da Presidência da República SEPPIR/PR. Brasília, DF.

Costa, V. G., Gomes, F. (2016). Religiões negras no Brasil: da escravidão à pós-emancipação. Selo Negro.

Crosby, J. W. \& Varela, J. G. (2014). Preferences for religious help-seeking: racial and gender differences, interfaith intolerance, and defensive theology. Mental Health, Religion \& Culture, [s. 1.], v. 17, n. 2, p. 196-209.

Da Motta Lody, R. G. (2006). O povo do santo: religião, história e cultura dos orixás, voduns, inquices e caboclos. MartinsFontes.

Doebler, S. (2015). Love thy neighbor? Relationships between religion and racial intolerance in Europe. Politics and Religion, v. 8 , n. 4 , p. 745-771.

Do Nascimento, W. F. (2016). Sobre os candomblés como modo de vida: Imagens filosóficas entre Áfricas e Brasis. Ensaios Filosóficos, 13 , $153-171$.

Du Plessis, L. (2009). Liberdade religiosa e igualdade como celebração da diferença: um desenvolvimento significativo na recente jurisprudência constitucional sulafricana. Potchefstroom Electronic Law Journal, v. 12, n. 4, p. 10-34, jan.

Eisinga, R., Billiet, J. \& Felling, A. (1999). Christian religion and ethnic prejudice in cross-national perspective. International Journal of Comparative Sociology, v. 40, n. 1, p. 375-393.

Ezeh, V. C. et al. (2017). Saliência de mortalidade e preconceito em relação às minorias etno-religiosas: Resultados e implicações de um estudo nigeriano. Journal of Psychology in Africa, v. 27, n. 5, p. 420-426.

Fanon, F. (2010). Os condenados da Terra. Minas Gerais: Editora UFJF.

Farias, J. B. (2006). Cidades negras: africanos, crioulos e espaços urbanos no Brasil escravista do século XIX. Alameda.

Gaia, R. D. S. P., da Silva Vitória, A., \& Scorsolini-Comin, F. (2021). Contribuições das Religiões de Matriz Africana para a Etnopsicologia brasileira. Revista Sociais e Humanas, 34(1).

GiorgI, A. \& SOUSA, D. (2010). Método fenomenológico de investigação em psicologia. Lisboa: Fim de século, p. 73-91.

Gonçalves, A. B. et al. (2011). Direitos Humanos e (in) tolerância religiosa: laicismo-proselitismo-fundamentalismo-terrorismo. São Paulo.

Guilliomet, D. M. (2020). Tolérance et lä̈cité.Philosophie. Academie de Normandie - perimetre Caen.

Ikram, U. Z. et al. (2016). Perceived ethnic discrimination and depressive symptoms: the buffering effects of ethnic identity, religion and ethnic social network. Social Psychiatry and Psychiatric Epidemiology, v. 51, n. 5, p. 679-688.

Law, R. (2006). Etnias de africanos na diáspora: novas considerações sobre os significados do termo'mina'. Tempo, v. 10, n. 20, p. 98-120.

Maggie, Y. (1992). Medo do feitiço: relações entre magia e poder no Brasil. In: Medo do feitiço: relações entre magia e poder no Brasil, p. $292-292$.

Mata, V. P. da \& Dos Santos, D. R. (2015). O papel do psicólogo numa política pública de combate a práticas racistas. Revista Brasileira de Psicologia, 02(núm. esp.), Salvador, Bahia.

Mccleary-gaddy, A. T. \& Miller, C. T. (2019). Negative religious coping as a mediator between perceived prejudice and psychological distress among African Americans: A structural equation modeling approach. Psychology of Religion and Spirituality, v. 11, n. 3, p. 257.

Mcewen, H. \& Steyn, M. (2016). Política de fé: Transformando comunidades religiosas e subjetividades espirituais na África do Sul pós-apartheid. Herv. teol. stud., Pretória, v. 72, n. 1, p. 1-8.

Munanga, K. (2015). Negritude-usos e sentidos. Autêntica. 
Research, Society and Development, v. 10, n. 11, e555101119634, 2021

(CC BY 4.0) | ISSN 2525-3409 |DOI: http://dx.doi.org/10.33448/rsd-v10i11.19634

Nascimento, R. N. A. (2015). Vozes da África nos simbolismos da ficção: ecos do preconceito étnico-religioso em Tenda dos Milagres. Comunicação Mídia e Consumo, v. 12 , n. 35, p. 12-30.

Nascimento, A. (2020). O quilombismo. Editora Perspectiva SA.

Nogueira, S. (2020). Intolerância religiosa. Pólen Produção Editorial LTDA.

ONU. (2019). Declaração de princípios sobre tolerância. Paris, 1995. http:// www.dhnet.org.br/sip/onu/paz/dec95.htm. Acesso em 10 jan.

Osborn, R. E. (2014). Does "Religious Violence" Exist?: An Argument Against Essentialism with Particular Reference to the Conquest of the Americas. Politics \& Religion, v. 7, n. 3.

Prandi, R. (2018). As religiões afro-brasileiras nas ciências sociais: uma conferência, uma bibliografia. Revista Brasileira de Informação Bibliográfica em Ciências Sociais BIB- ANPOCS, São Paulo, $\mathrm{n}^{\circ}$ 63, 2007, p. 7-30.

Prandi, R. (2000). De africano a afro-brasileiro: etnia, identidade, religião. Revista Usp, (46), 52-65.

Sanni, J. S. (2016). Religião: uma nova luta pela identidade africana. Phronimon, Pretória, v. 17, n. 2, p. 1-13.

Santos, B. S. (2014). Se Deus fosse um ativista dos direitos humanos. Cortez Editora, $2^{\mathrm{a}}$ edição.

Silva, L. C. \& Soares, K. R. A. (2015). A intolerância religiosa face às religiões de matriz africana como expressão das relações étnico-raciais brasileiras: o terreno do combate à intolerância no município de Duque de Caxias, 2015. Revista EDUC-Faculdade de Duque de Caxias, v. 01, nº 03, Jan-Jun.

Sodré, M. (2019). O terreiro e a cidade: a forma social negro-brasileira. Mauad Editora Ltda.

Sorkin, A. (2017). Debatendo liberdade religiosa e discriminação. Por John Corvino, Ryan T. Anderson, e Sherif Girgis. Nova Iorque: Oxford University Press,

Sousa, D. (2010). Método fenomenológico de investigação em psicologia. Lisboa: Fim de século, p. 73-91.

Viegas, F. (2016). A intolerância religiosa enquanto processo de destruição do outro. In: O que é intolerância religiosa? Lisboa: Escolar Editora, Cadernos de Ciências Sociais, p.13-42. 